\title{
Biocerâmicas aditivadas com nióbio (V): avaliação da rota hidrotérmica modificada com ácido cítrico e ureia para obtenção de hidroxiapatitas modificadas
}

\section{(Niobium (V) doped bioceramics: evaluation of the hydrothermal route modified with citric acid and urea to obtain modified hydroxiapatites)}

\author{
E. Simomukay, E. C. F. de Souza ${ }^{1,2}$, S. R. M. Antunes ${ }^{1,2}$, C. P. F. Borges ${ }^{1,2}$, M. D. Michel ${ }^{3}$, A. C. Antunes ${ }^{1,2}$ \\ ${ }^{1}$ Programa do Mestrado em Química Aplicada - UEPG \\ ${ }^{2}$ Laboratório de Química de Materiais \\ ${ }^{3}$ Laboratório de Engenharia de Materiais
}

Universidade Estadual de Ponta Grossa, Av. Carlos Cavalcanti, 4748, Uvaranas, Ponta Grossa, PR 84030-000

\begin{abstract}
Resumo
A hidroxiapatita sintética $\left(\mathrm{Ca}_{10}\left(\mathrm{PO}_{4}\right)_{6}(\mathrm{OH})_{2} ; \mathrm{HA}\right)$ devido à propriedade de biocompatibilidade com o tecido ósseo tornou-se um material cerâmico amplamente utilizado na reconstrução óssea. A biocompatibilidade assim como outras propriedades físico-químicas da hidroxiapatita podem ser modificadas por meio da adição de diferentes íons na sua estrutura. O íon nióbio (V) não tem sido empregado comumente na síntese de hidroxiapatitas. O objetivo deste trabalho foi verificar o emprego da rota hidrotérmica na síntese de hidroxiapatita dopada com íon nióbio (V). A rota empregada utiliza o complexo di-hidrogeno tris(oxalato) oxiniobato $(\mathrm{V})$ de amônio trihidratado $\left(\mathrm{NH}_{4} \mathrm{H}_{2}\left[\mathrm{NbO}\left(\mathrm{C}_{2} \mathrm{O}_{4}\right)_{3}\right] .3 \mathrm{H}_{2} \mathrm{O}\right)$, como precursor de íon nióbio (V). A adição de ácido cítrico e ureia na rota hidrotérmica é usada para o controle do $\mathrm{pH}$ da síntese e da taxa de precipitação. Amostras pura e aditivada com 5,3 ppm de íon nióbio (V) foram obtidas. A coexistência de outras fases além da hidroxiapatita não foi observada em ambas amostras por meio dos empregos das técnicas de difração de raios $\mathrm{X}$ e espectroscopia no infravermelho. Por meio da técnica de FTIR observou-se a presença de grupos funcionais característicos da hidroxiapatita. Na análise por microscopia eletrônica de varredura, verificou-se a formação de aglomerados compostos por partículas arredondadas confirmadas pela técnica de microscopia eletrônica de transmissão. A análise espectroscópica de fluorescência de raios X detectou a presença de nióbio na amostra aditivada obtida. Os resultados apontam que podem ser sintetizadas hidroxiapatitas aditivadas com íon nióbio (V) por meio da rota hidrotérmica, podendo ser considerado como enorme o potencial para a aplicação em biocerâmicas.

Palavras-chave: biomateriais, hidrotérmica, hidroxiapatita, nióbio.
\end{abstract}

Abstract

Synthetic hydroxyapatite $\left(\mathrm{Ca}_{10}\left(\mathrm{PO}_{4}\right)_{6}(\mathrm{OH})_{2}\right.$; $\left.\mathrm{HA}\right)$ has become a widely used ceramic material for bone reconstruction due to its biocompatibility with the bone tissue. This biocompatibility as well as other physical and chemical properties of the hydroxyapatite can be modified by the addition of different ions to its structure. Niobium $(V)$ ion has not been commonly used in the hydroxyapatite synthesis. The objective of this study was to evaluate the use of hydrothermal route in the niobium (V) doped hydroxyapatite synthesis. The route used the niobium ammonium oxalate $\left(\mathrm{NH}_{4} \mathrm{H}_{2}\left[\mathrm{NbO}\left(\mathrm{C}_{2} \mathrm{O}_{4}\right)_{3}\right] \cdot 3 \mathrm{H}_{2} \mathrm{O}\right)$ complex as a niobium $(V)$ ion precursor. The addition of citric acid and urea in the hydrothermal route is used for the control of synthesis $p H$ and precipitation rate. Pure sample and sample added with 5.3 ppm of niobium $(V)$ ion were prepared. The coexistence of other phases besides the hydroxyapatite was not observed in any of the samples through the use of X-ray diffraction and infrared spectroscopy (FTIR) techniques. The FTIR technique revealed the presence of hydroxyapatite characteristic functional groups. The scanning electron microscopy analysis showed the formation of agglomerates composed of round particles, confirmed by the transmission electron microscopy technique. The X-ray fluorescence spectroscopic analysis detected the presence of niobium in the doped sample. The results showed that niobium (V) doped hydroxyapatite can be synthesized by means of hydrothermal route, which may be considered as huge potential for future application in bioceramics.

Keywords: biomaterials, hydrothermal, hydroxyapatite, niobium.

\section{INTRODUÇÃO}

A hidroxiapatita sintética $\mathrm{Ca}_{10}(\mathrm{PO} 4)_{6}(\mathrm{OH})_{2}$ é um material que pertence à classe dos ortofosfatos de cálcio. A estrutura cristalina é normalmente hexagonal ou monoclínica com uma razão molar de $\mathrm{Ca} / \mathrm{P}$ igual a 1,67 , a qual se aproxima do valor da hidroxiapatita de origem biológica, por exemplo, a que está presente nos ossos do corpo humano [1]. A aplicabilidade de hidroxiapatitas sintéticas na área de biomateriais é amplamente conhecida e utilizada devido a sua excelente biocompatibilidade, bioatividade, atoxicidade e propriedades não-mutagênicas [2]. A estrutura 
da hidroxiapatita sintética permite que ocorram substituições catiônicas e aniônicas, sendo as substituições dos íons $\mathrm{Ca}^{2+} \mathrm{e}$ dos grupos $\mathrm{PO}_{4}^{3-}$ as mais comuns. Essas substituições podem afetar as propriedades físicas, químicas e biológicas, como a cristalinidade, tamanho do cristalito, porosidade, solubilidade, resistência mecânica, biocompatibilidade, entre outras [3]. A utilização de compostos de nióbio como elementos de substituição na estrutura da hidroxiapatita sintética é ainda bastante incomum e os seus efeitos sobre as propriedades do material formado ainda pouco esclarecidos. O nióbio na forma de seu principal óxido, o pentóxido de nióbio, $\mathrm{Nb}_{2} \mathrm{O}_{5}$, tem apresentado na literatura propriedades bioativas, possibilitando seu uso em revestimentos antialérgicos de endopróteses e também como carga de preenchimento em adesivos para dentística [4]. A adição do pentóxido de nióbio em hidroxiapatita através de moagem e sinterização tem resultado em compósitos com boa sinterabilidade e propriedades mecânicas com uma estrutura densificada com poros interconectados [5].

A morfologia da partícula obtida, a distribuição do tamanho de partícula, a composição química e o estado de aglomeração podem ser requisitos para uma determinada aplicação como biomaterial, devido à influência da composição dos precursores, $\mathrm{pH}$, pressão e temperatura na obtenção de ortofosfatos de cálcio [6]. Dentre os processos químicos, a síntese hidrotérmica pode ser uma alternativa para a obtenção de biocerâmicas aditivadas [7]. A síntese hidrotermal ou hidrotérmica ocorre com o uso de um solvente e de íons precursores submetidos a aquecimento em uma temperatura normalmente acima da temperatura de ebulição do solvente em uma autoclave fechada com uma pressão interna maior que a atmosférica. Nesta síntese, os mecanismos de nucleação, crescimento e envelhecimento podem ser controlados ajustando-se as necessidades requisitadas. $\mathrm{O}$ processo de complexação dos cátions precursores na síntese hidrotérmica com agentes quelantes como EDTA, ácido tartárico e cítrico são bastante comuns, conduzindo a uma maior homogeneidade na formação dos cristais devido ao controle na liberação de íons e possibilitando a obtenção de fases cristalinas e compostos monofásicos [8]. Devido à influência do $\mathrm{pH}$ na formação de ortofosfatos de cálcio, é possível a utilização de agentes de regulação de pH na síntese [9].

Este trabalho tem por objetivo verificar a síntese de hidroxiapatita dopada com íon nióbio (V) utilizada para biomateriais utilizando a rota hidrotérmica. Para isso, foi elaborada uma rota hidrotérmica modificada com ácido cítrico para regular o $\mathrm{pH}$ inicial da síntese em meio ácido e também provocar a complexação de íons cálcio (II) presentes na solução hidrotermal inicial, enquanto a ureia foi empregada para promover a elevação do $\mathrm{pH}$ para meio básico durante a síntese hidrotérmica e, consequentemente, promover a precipitação da hidroxiapatita. Com a intenção de testar o uso de íon nióbio (V) em hidroxiapatitas, o complexo dihidrogeno tris(oxalato) oxiniobato (V) de amônio trihidratado foi introduzido em baixa concentração visando a possibilidade de se sintetizar hidroxiapatitas modificadas com este íon.

\section{MATERIAIS E MÉTODOS}

Preparo das amostras: amostra de hidroxiapatita sintética pura, HA, foi preparada utilizando-se solução aquosa de etanoato de cálcio monohidratado $\left(\mathrm{Ca}\left(\mathrm{CH}_{3} \mathrm{COO}\right)_{2}\right.$, Vetec) com concentração de $0,0835 \mathrm{~mol} / \mathrm{L}$ e solução aquosa 0,0501 $\mathrm{mol} / \mathrm{L}$ de monofosfato de amônio anidro - MAP $\left(\mathrm{NH}_{4} \mathrm{H}_{2} \mathrm{PO}_{4}\right.$, Ecibra-Cetus). A amostra aditivada com o precursor de nióbio, HA-Nb, foi preparada a partir de solução aquosa do complexo di-hidrogeno tris(oxalato) oxiniobato $(\mathrm{V}) \mathrm{de}$ amônio trihidratado - CAN $\left(\mathrm{NH}_{4} \mathrm{H}_{2}\left[\mathrm{NbO}\left(\mathrm{C}_{2} \mathrm{O}_{4}\right)_{3}\right] \cdot 3 \mathrm{H}_{2} \mathrm{O}\right.$, CBMM) com concentração de $57,6 \mathrm{mg} / \mathrm{L}$ do precursor correspondente a 5,3 mg/L de íon nióbio (V).

Rota da síntese utilizada: para o preparo da solução hidrotérmica, $50 \mathrm{~mL}$ da solução do precursor de cálcio foi acidificado até pH 4,0 com a adição do ácido 2-hidroxi-1,2,3 propanotricarboxílico monohidratado $\left(\mathrm{C}_{6} \mathrm{H}_{8} \mathrm{O}_{7} \cdot \mathrm{H}_{2} \mathrm{O}\right.$, Vetec), que também serviu como agente de complexação. Em seguida, adicionou-se lentamente $50 \mathrm{~mL}$ da solução do precursor de fósforo, com e sem a adição dos íons de nióbio (V). Adicionou-se a ureia como agente de controle do $\mathrm{pH}$ e transferiu-se a solução final obtida a uma autoclave de teflon, que foi levada a estufa pelo período de $24 \mathrm{~h}$ a $165^{\circ} \mathrm{C}$. Ao final deste período, foi medido o $\mathrm{pH}$ final da solução hidrotermal obtida, situando-se na faixa entre 9 a 10, e procedeu-se em seguida à lavagem com água deionizada até $\mathrm{pH} 7$, seguido de filtração simples e secagem do pó obtido em estufa de circulação de ar quente a $110^{\circ} \mathrm{C}$. As soluções resultantes da filtração simples foram rotuladas e armazenadas em frasco de vidro âmbar para posterior descarte.

Caracterizações físico-químicas: os pós obtidos foram caracterizados através da análise por difração de raios $\mathrm{X}$ (DRX) utilizando-se um difratômetro de raios X Shimadzu XRD-6000, a fim de determinar as fases cristalinas presentes. Os difratogramas obtidos foram comparados com dados encontrados nos bancos de dados do Crystallography Open Database (COD) [10]. Foram realizadas medidas de espectroscopia de absorção na região do infravermelho por transformada de Fourier (FTIR) utilizando um espectrofotômetro FTIR Shimadzu IR Prestigie 21 em uma faixa de leitura de $400 \mathrm{a} 4000 \mathrm{~cm}^{-1}$ para se determinar os grupos funcionais presentes nas amostras. A análise da morfologia dos pós obtidos foi realizada utilizando um microscópio eletrônico de varredura Shimadzu - SS 550 com sonda EDX. As amostras foram dispersas em álcool isopropílico e depositadas nos porta-amostras e depois de secas foram metalizadas em evaporadora Shimadzu para deposição de ouro para facilitar a análise microscópica e visualização do formato das partículas. A análise de microscopia eletrônica de transmissão foi realizada utilizando um microscópio eletrônico de transmissão Phillips C-20 com constante de câmera de 1224 pixel x angstrom $(\AA)$. As amostras foram submetidas à secagem em estufa por $24 \mathrm{~h}$ a $110^{\circ} \mathrm{C}$, acondicionadas em eppendorf e armazenadas em dessecador até ser encaminhada para a análise. As micrografias foram analisadas com o programa ImageJ e, para indexação dos planos cristalinos, os raios dos halos (em pixels) foram 
medidos por três vezes com o programa Diffraction Ring Profiler for Windows v.1.7, sendo que a distância interplanar $\left(\mathrm{d}_{\mathrm{hkl}}\right)$ foi calculada usando-se a Equação A:

$\mathrm{d}_{\mathrm{hkl}}=$ constante da câmera/raio do círculo $(\AA)$

O pó aditivado foi caracterizado por espectroscopia de fluorescência de raios X. Os teores dos elementos foram determinados por análise sem padrões (standarless) de elementos químicos do flúor até o urânio em espectrômetro de fluorescência de raios X PANalytical Axios Advanced. Os resultados foram expressos em \% de óxido em base calcinada normalizada a $100 \%$.

\section{RESULTADOS E DISCUSSÃO}

Através da técnica de difração de raios $\mathrm{X}$ foram obtidos difratogramas apresentados na Fig. 1; em todas as amostras nota-se a concordância e predominância da fase de hidroxiapatita, segundo a comparação com o padrão do banco de dados COD 1011242 (pontilhado no difratograma da amostra HA).

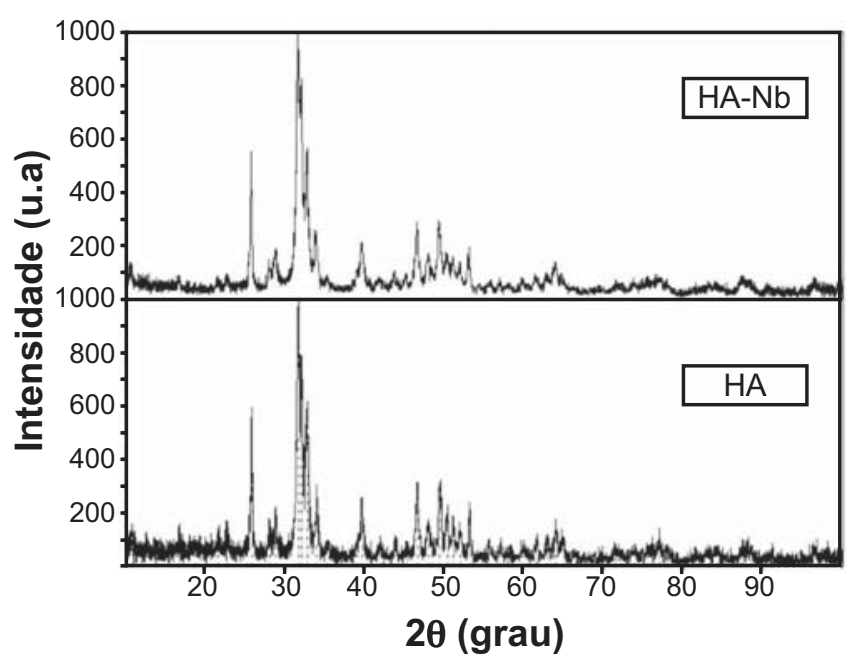

Figura 1: Difratogramas de raios $\mathrm{X}$ das amostras HA e HA$\mathrm{Nb}$ obtidas por síntese hidrotérmica. As linhas verticais (|) correspondem ao padrão de hidroxiapatita COD 1011242.

[Figure 1: X-ray diffraction patterns of the samples $\mathrm{HA}$ and $\mathrm{HA}-\mathrm{Nb}$ after hydrothermal synthesis. The vertical lines (|) are from COD 1011242 pattern of hydroxyapatite.]

Para a amostra sem aditivação (HA), considerando-se os precursores de cálcio e fósforo empregados e os resultados obtidos, tem-se possivelmente a reação da síntese segundo a Equação B:

$$
\begin{aligned}
& 10 \mathrm{Ca}\left(\mathrm{CH}_{3} \mathrm{COO}\right)_{2(\mathrm{aq})}+6\left(\mathrm{NH}_{4}\right) \mathrm{H}_{2} \mathrm{PO}_{4(\mathrm{aq})}+14 \mathrm{OH}^{-}{ }_{(\mathrm{aq})} \rightarrow \\
& \mathrm{Ca}_{10}\left(\mathrm{PO}_{4}\right)_{6}(\mathrm{OH})_{2(\mathrm{~s})}+6 \mathrm{NH}_{4}^{+}{ }_{(\mathrm{aq})}+20 \mathrm{CH}_{3} \mathrm{COO}_{(\mathrm{aq})}^{-}+12 \mathrm{H}_{2} \mathrm{O}_{(\mathrm{l})}
\end{aligned}
$$

Esta possível reação química mantém a razão atômica $\mathrm{Ca} / \mathrm{P}$ igual a 1,67, justificando a concordância da fase cristalina da amostra HA com o padrão COD 1011242. $\mathrm{Na}$ amostra HA-Nb que teve a dopagem de 5,76 ppm do complexo amoniacal de nióbio (CAN) é plausível que íons de
$\mathrm{Nb}(\mathrm{V})$ possam se incorporar na estrutura da hidroxiapatita sem a formação de outras fases, permanecendo na estrutura da hidroxiapatita sem a formação de fases contendo nióbio, conforme visto nos resultados da difração de raios $\mathrm{X}$.

Tem sido sugerido que o monômero aniônico $\mathrm{H}_{4} \mathrm{NbO}_{6}^{3-}$ é mais favorávelà incorporação substitucional na hidroxiapatita nas posições do grupo $\mathrm{PO}_{4}^{3-}$, devido à semelhança nos raios iônicos e à estabilidade eletrônica [11]. Levando-se em conta o $\mathrm{pH}$ da síntese utilizado neste trabalho e a literatura citada, as espécies trivalentes como o ânion $\left[\mathrm{NbO}_{2}(\mathrm{OH})_{4}\right]^{3-}$ e $\mathrm{H}_{4} \mathrm{NbO}_{6}{ }^{3-} \mathrm{e}$ até espécies monovalentes como o oxoaníon $\mathrm{NbO}_{3}^{-}$poderiam estar presentes e ocasionar uma possível substituição em sítios da hidroxila, sendo uma explicação plausível para a possibilidade de substituição, assim como ocorre nas substituições amplamente conhecidas de grupos fosfatos com grupos carbonatos.

A complexidade química em solução aquosa do CAN, com a possibilidade de surgimento de diversas espécies químicas durante o processo hidrotérmico que induz a hidrólise de seus componentes, dificulta a avaliação inicial dos resultados. Uma possível e suposta reação substitucional na amostra aditivada com 5,76 ppm do precursor de nióbio nos sítios de grupos fosfatos $\mathrm{PO}_{4}^{3-}$ poderia ser proposta como a reação da Equação C:

$$
\begin{aligned}
& \mathrm{Ca}\left(\mathrm{CH}_{3} \mathrm{COO}\right)_{2(\mathrm{aq})}+\left(\mathrm{NH}_{4}\right) \mathrm{H}_{2} \mathrm{PO}_{4(\mathrm{aq})}+\mathrm{OH}_{(\mathrm{aq})}^{-}+\mathrm{H}_{4} \mathrm{NbO}_{6}^{3-}{ }_{(\mathrm{aq})}^{-} \rightarrow \\
& \mathrm{Ca}_{10}\left(\mathrm{PO}_{4}\right)_{6-\mathrm{x}}\left(\mathrm{H}_{4} \mathrm{NbO}_{6}^{3-}\right)_{\mathrm{x}}(\mathrm{OH})_{2(\mathrm{~s})}+\mathrm{NH}_{4}^{+}{ }_{(\mathrm{aq})}+\mathrm{CH}_{3} \mathrm{COO}_{(\mathrm{aq})}^{-}+\mathrm{H}_{2} \mathrm{O}_{(\mathrm{l})}
\end{aligned}
$$

A incógnita $\mathrm{x}$ é um valor estequiométrico que varia de acordo com o grau de substituição dos grupos fosfatos. Para uma substituição nos sítios de hidroxila $\mathrm{OH}^{-}$, tem-se a possível reação da Equação D:

$$
\begin{aligned}
& \mathrm{Ca}\left(\mathrm{CH}_{3} \mathrm{COO}\right)_{2(\mathrm{aq})}+\left(\mathrm{NH}_{4}\right) \mathrm{H}_{2} \mathrm{PO}_{4(\mathrm{aq})}+\mathrm{OH}_{(\mathrm{aq})}^{-}+\mathrm{NbO}_{3(\mathrm{aq})}^{-} \rightarrow \\
& \mathrm{Ca}_{10}\left(\mathrm{PO}_{4}\right)_{6}(\mathrm{OH})_{2-\mathrm{x}}\left(\mathrm{NbO}_{3}\right)_{\mathrm{x}(\mathrm{s})}+\mathrm{NH}_{4(\mathrm{aq})}^{+}+\mathrm{CH}_{3} \mathrm{COO}_{(\mathrm{aq})}^{-}+\mathrm{H}_{2} \mathrm{O}_{(\mathrm{l})}^{(\mathrm{D})}
\end{aligned}
$$

No entanto, é possível até a ocorrência de ambas as substituições nos grupos fosfato e hidroxila, assim como ocorre nas substituições dos grupos carbonatos [12], como também o aparecimento de outras espécies contendo nióbio, o que leva a necessidade de estudos posteriores para elucidação do mecanismo de formação dos compostos nesta rota hidrotérmica.

Analisando os dois difratogramas das duas amostras não foi encontrada correlação com outras fases cristalinas de ortofosfatos de cálcio plausíveis de se formarem durante a síntese, como o fosfato de cálcio hidratado $\left(\mathrm{Ca}_{3}\left(\mathrm{PO}_{4}\right)_{2} \cdot \mathrm{xH}_{2} \mathrm{O}\right)$, fosfato de cálcio pentahidratado, $\left(\mathrm{Ca}_{8} \mathrm{H}_{2}\left(\mathrm{PO}_{4}\right)_{6} .5 \mathrm{H}_{2} \mathrm{O}\right)$, fosfato tetracálcico $\left(\mathrm{Ca}_{4} \mathrm{P}_{2} \mathrm{O}_{9}\right)$ e fosfato dicálcio dihidratado $\left(\mathrm{CaHPO}_{4} \cdot 2 \mathrm{H}_{2} \mathrm{O}\right)$, e pode-se creditar ao uso da rota hidrotérmica modificada com controle da faixa de $\mathrm{pH}$ de precipitação que possibilita a precipitação preferencial da hidroxiapatita.

A análise espectroscópica FTIR foi feita com objetivo de confirmar as fases identificadas na análise por DRX sendo que os espectros de absorção na região do infravermelho estão apresentados na Fig. 2. A amostra HA apresentou os principais modos de absorção característicos da 

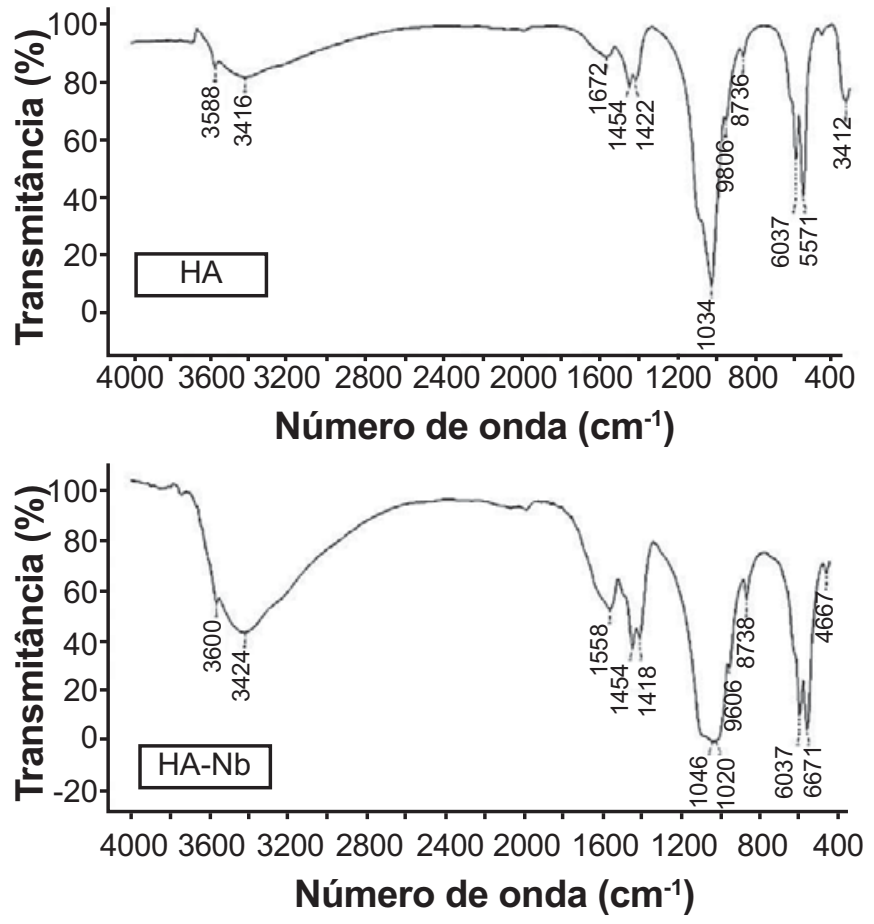

Figura 2: Espectros de infravermelho das amostras HA e HA-Nb entre 400-4000 $\mathrm{cm}^{-1}$.

[Figure 2: Infrared spectra of the samples $\mathrm{HA}$ and $\mathrm{HA}-\mathrm{Nb}$ between $400-4000 \mathrm{~cm}^{-1}$.]

hidroxiapatita, representados pelas bandas com número de onda em $3568,3416,1572,1454,1422,1034,960,6,873,8$, $603,7,567,1$ e $347,2 \mathrm{~cm}^{-1}$. A banda em $3568 \mathrm{~cm}^{-1}$ foi atribuída ao grupo hidroxila $\mathrm{OH}^{-}$estrutural, $3416 \mathrm{~cm}^{-1}$ à presença de $\mathrm{H}_{2} \mathrm{O}, 1572 \mathrm{~cm}^{-1}$ à substituição do grupo $\mathrm{OH}^{-}$por um grupo $\mathrm{CO}_{3}^{2-}$, e as bandas em 1454,1422 e $873,8 \mathrm{~cm}^{-1}$ atribuídas ao grupo carbonato $\mathrm{CO}_{3}^{2-}$, que está provavelmente substituindo grupos fosfatos $\mathrm{PO}_{4}^{3-}$, enquanto a banda em $1034 \mathrm{~cm}^{-1}$ foi atribuída ao grupo $\mathrm{PO}_{4}^{3-}$ no modo dobramento v3, em 960,6 $\mathrm{cm}^{-1}$ ao grupo $\mathrm{PO}_{4}^{3-} \mathrm{em}$ estiramento $v 1$, em $603,7 \mathrm{~cm}^{-1}$ ao grupo $\mathrm{PO}_{4}^{3-}$ em modo dobramento $v 4$, em $567,1 \mathrm{~cm}^{-1}$ ao grupo $\mathrm{PO}_{4}{ }^{4-}$ - em modo dobramento $v 4 \mathrm{e}$ a $347,2 \mathrm{~cm}^{-1}$ ao grupo hidroxila.

A amostra aditivada com 5,76 ppm do CAN mostrou os principais modos de absorção característicos da hidroxiapatita e praticamente o mesmo resultado que a amostra não aditivada, provavelmente devido à baixa quantidade de aditivação. Observaram-se as bandas com número de onda em 3566, 3424, 1568, 1454, 1418, 1045, $1028,960,6,873,8,603,7,567,1$ e $468,7 \mathrm{~cm}^{-1}$, sendo a banda em $3566 \mathrm{~cm}^{-1}$ assinalada ao grupo hidroxila $\mathrm{OH}^{-}$estrutural, $3424 \mathrm{~cm}^{-1}$ à presença de $\mathrm{H}_{2} \mathrm{O}, 1454 \mathrm{e} 1418 \mathrm{~cm}^{-1}$ atribuídas à substituição tipo B do grupo carbonato $\mathrm{CO}_{3}{ }^{2-}, 1045$ e 1028 $\mathrm{cm}^{-1}$ ao grupo $\mathrm{PO}_{4}^{3-}$ no modo dobramento $v 3$, em $960,6 \mathrm{~cm}^{-1}$ ao grupo $\mathrm{PO}_{4}^{3-} \mathrm{em}$ estiramento $v 1$, em $603,7 \mathrm{~cm}^{-1}$ ao grupo $\mathrm{PO}_{4}{ }^{3-}$ no modo dobramento $v 4$, em $567,1 \mathrm{~cm}^{-1}$ ao grupo $\mathrm{PO}_{4}^{3-}$ no modo dobramento $v 4$ e uma banda em $472,6 \mathrm{~cm}^{-1}$ ao dobramento $v 2$ do grupo $\mathrm{PO}_{4}^{3-}$. Uma banda em $1568 \mathrm{~cm}^{-1}$ indica a substituição do grupo hidroxila $\mathrm{OH}^{-}$pelo grupo carbonato $\mathrm{CO}_{3}^{2-}$, de modo que provavelmente tenha ocorrido uma substituição do tipo $\mathrm{AB}$ na rede cristalina das amostras. A substituição do tipo $A B$ provoca uma diminuição da cristalinidade das amostras e alterações nos parâmetros cristalinos da amostra.

Os resultados da caracterização por espectroscopia de absorção do infravermelho concordaram com os valores encontrados na literatura [13]. No estudo de hidroxiapatitas nanocarbonatadas obtidas por síntese hidrotérmica, foi creditado ao íon carbonato $\mathrm{CO}_{3}^{2-} \mathrm{o}$ aumento do parâmetro de rede $a$ e a diminuição do parâmetro de rede $c$, quando ocorre a substituição do tipo $\mathrm{A}$, e a diminuição do parâmetro de rede $a$ e o aumento do parâmetro de rede $c$, quando da substituição do tipo $\mathrm{B}$, pelo fato do comprimento da ligação $\mathrm{P}-\mathrm{O}$ ser menor que o comprimento da ligação C-O [14]. O uso do MAP como precursor de fósforo poderia originar fosfatos de cálcio, como a hidroxiapatita deficiente em cálcio (CDHA), devido à dissociação do sal em solução aquosa que libera os íons de $\mathrm{HPO}_{4}^{2-}$, assim como a formação do fosfato de octacálcio (OCP, $\left.\mathrm{Ca}_{8}\left(\mathrm{HPO}_{4}\right)_{2}\left(\mathrm{PO}_{4}\right)_{4} \cdot 5 \mathrm{H}_{2} \mathrm{O}\right)$. Em estudo de decomposição da ureia para precipitação da hidroxiapatita, são citadas reações de transformação do OCP em hidroxiapatita, como apresentadas nas Equações E e F [15]:

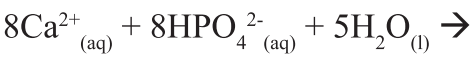

$$
\begin{aligned}
& \mathrm{Ca}_{8} \mathrm{H}_{2}\left(\mathrm{PO}_{4}\right)_{6}\left(\mathrm{H}_{2} \mathrm{O}\right)_{5(\mathrm{~s})}+2 \mathrm{H}_{3} \mathrm{PO}_{4(\mathrm{aq})} \\
& \mathrm{Ca}_{8} \mathrm{H}_{2}\left(\mathrm{PO}_{4}\right)_{6}\left(\mathrm{H}_{2} \mathrm{O}\right)_{5(\mathrm{~s})}+8 \mathrm{H}_{2} \mathrm{O}_{(\mathrm{l})} \rightarrow \\
& 4 \mathrm{Ca}_{10}\left(\mathrm{PO}_{4}\right)_{6}(\mathrm{OH})_{2(\mathrm{~s})}+6 \mathrm{H}_{3} \mathrm{PO}_{4(\text { aq })}
\end{aligned}
$$

Isto sugere uma possível formação de compostos metaestáveis durante a síntese hidrotérmica, que poderiam ocasionar a formação de outros ortofosfatos de cálcio como o OCP e CDHA, mas, segundo a análise da difração de raios $\mathrm{X}$ e espectroscopia no infravermelho, mesmo que tenha ocorrida a formação de outros compostos, a transformação em função do tempo de síntese hidrotérmica $(24 \mathrm{~h})$ foi completa para a fase de hidroxiapatita, em função da estabilidade desta fase no $\mathrm{pH}$ final da síntese, atingido após a decomposição da ureia.

Através da análise de espectroscopia por fluorescência de raios X (FRX), confirmou-se definitivamente a presença de nióbio na amostra HA-Nb. A análise química obtida através da fluorescência de raios X para esta amostra está apresentada na Tabela I. A análise foi realizada de forma semiquantitativa devido à pouca quantidade de amostra disponível e foi realizada sem padrões o que torna possível apenas a confirmação da presença do nióbio na amostra.

Tabela I - Análise elementar obtida por frx da amostra HA-Nb. [Table I - Elemental analysis from FRX of sample HA-Nb.]

\begin{tabular}{ccc}
\hline Óxido & \% em massa & \% atômica \\
\hline $\mathrm{CaO}$ & 62,00 & 5,885 \\
$\mathrm{P}_{2} \mathrm{O}_{5}$ & 37,80 & 2,835 \\
$\mathrm{Nb}_{2} \mathrm{O}_{5}$ & 0,20 & 0,008 \\
\hline
\end{tabular}


É possível uma verificação que a razão atômica entre $\mathrm{Ca} /$ $(\mathrm{Nb}+\mathrm{P})$ está próxima a 2,0, o que pode indicar a formação de uma hidroxiapatita modificada em relação à razão atômica de 1,67 da hidroxiapatita pura.

A Fig. 3 apresenta os resultados morfológicos das amostras obtidas pela técnica de microscopia eletrônica de varredura. A amostra HA apresentou uma morfologia com predominância de aglomerados formados por placas irregulares (Figs. 3A e 3B), enquanto na amostra HA$\mathrm{Nb}$ a morfologia dominante é a formação de aglomerados arredondados (Figs. 3C e 3D). Examinando a morfologia da amostra HA-Nb por microscopia eletrônica de transmissão (Figs. 4C e 4D), nota-se que o aglomerado é composto pela união de outros aglomerados menores. O aglomerado da Fig. 4C apresentou um diâmetro médio equivalente de $190 \mathrm{~nm}$. Estes aglomerados (Fig. 4D) são formados por partículas de tamanho nanométrico com diâmetro médio de $17 \mathrm{~nm}$ com formato predominante de bastonete (rod). As análises no programa ImageJ forneceram para o aglomerado da Fig.

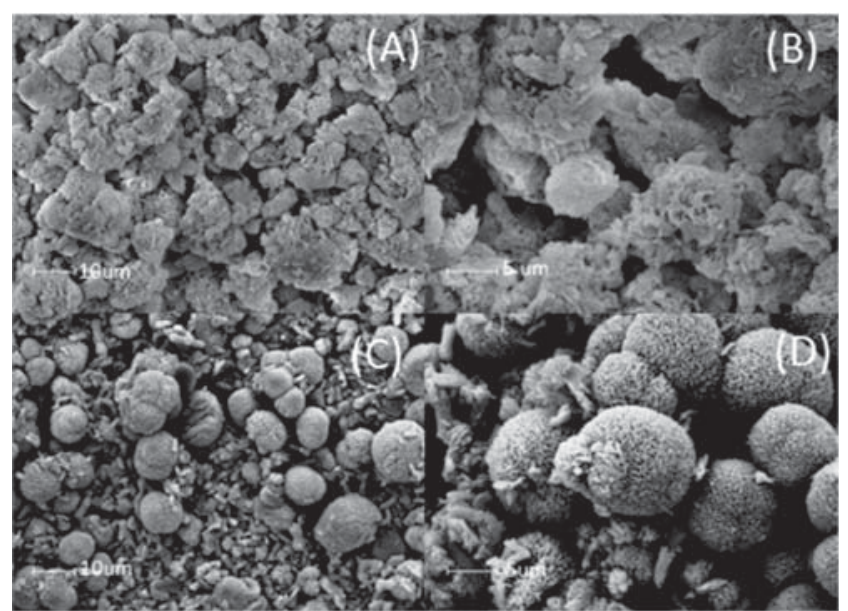

Figura 3: Micrografias obtidas por microscopia eletrônica de varredura das amostras: (A, B) HA; e (C, D) HA-Nb.

[Figure 3: SEM micrographs of the samples: $(A, B) H A$; and $(C$, D) $\mathrm{HA}-\mathrm{Nb}$.]
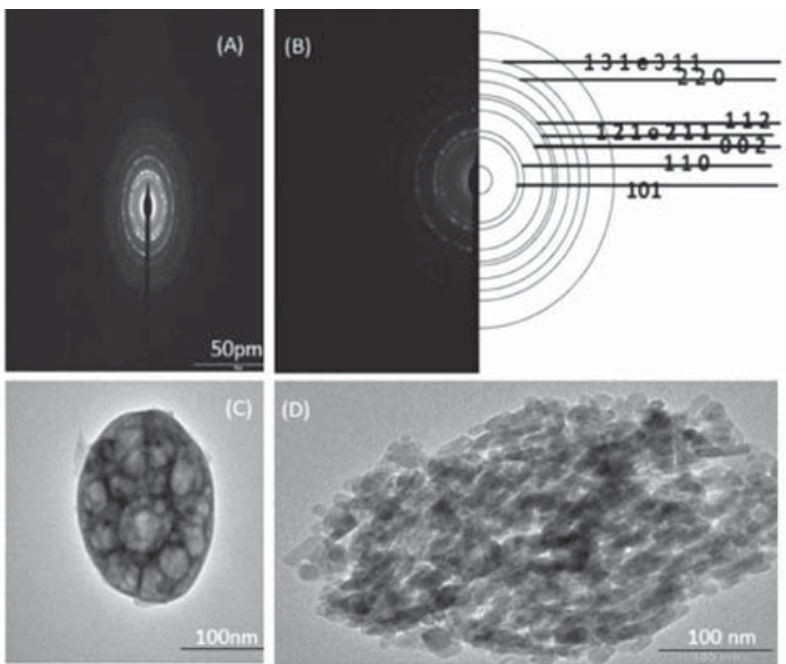

Figura 4: Micrografias obtidas em microscópio eletrônico de transmissão da amostra HA-Nb: (A) padrão de difração de elétrons de área selecionada; (B) indexação do padrão de difração de elétrons; (C) aglomerado arredondado formado; e (D) partículas não-aglomeradas da amostra.

[Figure 4: TEM micrographs of the sample HA-Nb: (A) selected area electron diffraction pattern; (B) indexing of electron diffraction pattern; $(C)$ rounded agglomerated of particles; and (D) non-agglomerated particles of sample.]

4C uma circularidade de 0,893 devido à irregularidade da forma e uma convexidade de 0,926 , o que indica um sólido compacto e relativamente regular com algum espaçamento superficial. Segundo resultados reportados [15], as partículas de hidroxiapatita com formatos de placas, bastões e fibras possuem maior área superficial e melhor adsorção molecular que outros materiais como sílica, quantum dots e nanotubos de carbono, sendo citado que estes formatos potencializam o seu uso em compósitos de biomateriais como cimentos ósseos, que exigem melhor resistência mecânica, e desta forma há uma clara potencialidade de aplicações em biomateriais para o material obtido neste trabalho com a aditivação de íon nióbio.

Tabela II - Indexação dos halos do padrão de difração de elétrons da amostra HA-Nb. [Table II - Indexing SAED pattern of HA-Nb sample.]

\begin{tabular}{cccccc}
\hline Halo & $\begin{array}{c}\text { Raio médio do } \\
\text { halo (pixel) }\end{array}$ & $\begin{array}{c}\text { Constante da câmera } \\
\text { (pixel. } \AA \text { ) }\end{array}$ & $\begin{array}{c}\text { Distância } \\
\text { interplanar } \\
\text { calculada, } \mathrm{d}(\AA)\end{array}$ & $\begin{array}{c}\mathrm{d}_{\text {hkl }} \text { do padrão de } \\
\text { hidroxiapatita* } \\
(\AA)\end{array}$ & $\begin{array}{c}\text { Planos hk1 } \\
\text { correspondentes } \\
\text { ao padrão de } \\
\text { hidroxiapatita* }\end{array}$ \\
\hline R1 & 228,90 & 1224 & 5,347 & 5,263 & 101 \\
R2 & 259,43 & 1224 & 4,718 & 4,716 & 110 \\
R3 & 355,26 & 1224 & 3,445 & 3,440 & 002 \\
R4 & 433,59 & 1224 & 2,822 & 2,817 & 121 ou 211 \\
R5 & 440,37 & 1224 & 2,779 & 2,779 & 112 \\
R6 & 519,03 & 1224 & 2,358 & 2,358 & 220 \\
R7 & 568,31 & 1224 & 2,153 & 2,152 & 131 ou 311 \\
\hline *-501568
\end{tabular}

*-501568 Base de Dados Crystmet. 
Aditivos, como citrato de sódio, polietileno glicol, EDTA, PVA e dendrímeros, têm sido bastante empregados para controle da morfologia, originando micro e nanofibras, hastes, elipsoides e outras morfologias. O EDTA, por exemplo, atua como um agente quelante para os íns de cálcio (II) e evita a formação de DCPD e OCP, resultando em uma diminuição da nucleação da hidroxiapatita e o crescimento ao longo do eixo "c", o que provoca um alongamento da partícula e leva à formação de partículas tipo whisker [16]. A presença nesta rota hidrotérmica de elementos capazes de promover uma maior complexação dos íons $\mathrm{Ca}(\mathrm{II})$ e $\mathrm{Nb}(\mathrm{V})$ pode ter influenciado na morfologia arredondada e aglomerada das partículas, resultando em uma maior organização morfológica nas amostras com nióbio (V) do que na amostra não aditivada HA.

O padrão de difração de elétrons obtido por microscopia eletrônica de transmissão (Fig. 4A) mostra a formação de halos que indicam a policristalinidade da amostra HA$\mathrm{Nb}$. Os resultados da indexação destes halos podem ser observados na Fig. 4B e na Tabela II.

Observa-se a correlação dos valores de distância interplanar $\left(\mathrm{d}_{\mathrm{hkl}}\right)$ calculados a partir dos raios medidos dos halos de difração com os da hidroxiapatita, conforme dados da base cristalográfica Crystmet 501568, e a identificação dos respectivos planos cristalográficos (hkl). A distância interplanar de 2,817 $\AA$ é relacionada aos planos (121) e (211), sendo este plano o de maior intensidade ( $\mathrm{I}=100)$; o mesmo ocorre com a distância interplanar de 2,152 A, em que o plano de maior intensidade é o plano (311) de intensidade igual a 8. Levando-se em conta a solubilidade de fosfatos de cálcio e as condições da síntese ao qual partiu de um meio ácido, onde a fase de hidroxiapatita é instável, a tendência inicial seria a possível formação de fosfato de cálcio amorfo com posterior transformação para a fase de hidroxiapatita durante a realização da síntese. Portanto, pode-se averiguar pelo resultado que o uso de um tempo longo de síntese (24 h) e o pH final adotado permitiu que uma possível formação de fosfato de cálcio amorfo proporcionasse a transformação na fase cristalina de hidroxiapatita, o que demonstra a concordância com os resultados da difração de raios X.

\section{CONCLUSÃO}

A adição de nióbio na síntese hidrotérmica de hidroxiapatita sintética na proporção de $5,2825 \mathrm{ppm}$ de íon nióbio (V) aparentemente não resultou na formação de outras fases, como óxidos de nióbio e niobatos de cálcio. O desempenho das hidroxiapatitas modificadas ou não está muito relacionada à morfologia, ao tamanho da partícula e a estruturação dimensional [17] e neste trabalho pode-se verificar estas influências. $\mathrm{O}$ uso de um precursor de íon nióbio (V) para obtenção de hidroxiapatita modificada foi possível com o uso da rota hidrotérmica alterada com aditivos para controle da taxa de precipitação e pH final da síntese.
Trabalhos futuros atentam para o estudo de concentrações mais elevadas e estudos de citocompatibilidade que caracterizem a amostra obtida como um material bioativo para uso em reparo e regeneração óssea, com estruturas esféricas e porosas que favorecem o carregamento e liberação de fármacos e genes.

\section{AGRADECIMENTOS}

Os autores agradecem ao apoio financeiro da CAPES/PNPD, CNPq, Fundação Araucária e às análises realizadas pelos laboratórios: Complexo de Laboratórios Multiusuários (C-LABMU) da UEPG, Laboratório Interdisciplinar de Microscopia do curso de Engenharia de Materiais da UEPG, Grupo de Física Aplicada a Solos e Ciências Ambientais do Departamento de Física da UEPG e Laboratórios de Microscopia do Instituto de Química UNESP Araraquara - SP.

\section{REFERÊNCIAS}

[1] G. Ma, X.Y. Liu, Cryst. Growth Des. 9, 7 (2009) 2991.

[2] E.C.C. Reis, Braz. Arch. Biol. Technol. 53, 4 (2010) 817.

[3] D.M. Ibrahim, A.A. Mostafa, S.I. Korowash, Chem. Cent. J. 5 (2011) 74.

[4] V.C. Leitune, F.M. Collares, A. Takimi, G.B. Lima, C.L. Petzhold, C.P. Bergmann, S.M. Samuel, J. Dent. 41, 2 (2013) 106.

[5] W.J. Nascimento, T.G.M. Bonadio, V.F. Freitas, W.R. Weinand, M.L. Baesso, M.W. Lima, Mater. Chem. Phys. 130 (2011) 84.

[6] J. Liu, X. Ye, H. Wang, Ceram. Int. 29, 6 (2003) 629.

[7] X.Y. Zhao, Y.J. Zhu, F. Chen, B.Q. Lu, C. Qi, J. Zhao, J. Wua, Cryst. Eng. Comm. 15 (2013) 7926.

[8] A.K. Nayak, Int. J. Chem. Tech. Res. 2, 2 (2010) 903.

[9] I.Y. Kim, K. Kikuta, C. Ohtsuki, Mater. Sci. Eng. C 31, 7 (2011) 1383.

[10] COD, Crystallographic Open Database, URL: $<$ http:// sdpd.univ-lemans.fr/cod/>.

[11] M. Tamai, K.J. Isama, J. Kazuo, J. Artif. Organs 10 (2007) 22.

[12]T. Toru, T. Ikoma, T. Yoshioka, H. Nobutaka, T. Junzo, J. Mater. Sci. 45 (2010) 2419.

[13] A.F. Khan, M. Awais, A.S. Khan, S. Tabassum, A.A. Chaudhry, I.Ur Rehman, Appl. Spectrosc. Rev. 48, 4 (2013) 329.

[14] X. Xiao, R. Liu, Q. Huang, X. Ding, J. Mater. Sci. Matter. Med. 20, 12 (2012) 2375.

[15] W. Xia, C. Lindahl, C. Persson, P. Thomsen, J. Lausmaa, H. Engovist, J. Biomater. Nanobiotechnol. 1 (2010) 7.

[16] Z. Qinfu, Appl. Surf. Sci. 257, 23 (2011) 10126.

[17] C. Chen, Z. Huang, W. Yuan, J. Li, C. Xiaokun, C. Ruan, Cryst. Eng. Comm. 13 (2011) 1632.

(Rec. 03/10/2014, Rev. 22/09/2015, Ac. 02/10/2015) 